\title{
Observations of pulsations in roAp stars
}

\author{
O. Kochukhov \\ Department of Astronomy and Space Physics, Uppsala University, \\ Box 515, SE-751 20 Uppsala, Sweden
}

\begin{abstract}
I review recent results of the observations of magnetoacoustic p-mode oscillations in roAp stars with the focus on time-resolved spectroscopic studies. Time-series spectroscopy of roAp stars reveals unexpected and diverse pulsational behaviour in the spectral lines of different chemical elements. These unique pulsational characteristics arise from an interplay between the short vertical length of pulsation waves and extreme chemical stratification in the atmospheres of peculiar stars. This enables a tomographic reconstruction of the depth-dependence of the chemical composition and pulsation wave properties. The combination of magnetoacoustic tomography with Doppler imaging of the horizontal non-radial pulsation pattern opens the possibility for an unprecedented three-dimensional mapping of roAp star atmospheres.
\end{abstract}

\section{Introduction}

A significant fraction of the upper main sequence stars of spectral classes between $B$ and $F$ possesses a strong, ordered magnetic field and shows a surface chemical composition strongly deviating from that of the Sun. These chemically peculiar $\mathrm{Ap} / \mathrm{Bp}$ stars are characterized by unusually slow rotation and they show spectacular variability of the field strength, mean brightness and spectra on the rotation time scale. This is understood to be a result of the rotational modulation of the aspect at which the stable stellar magnetic field geometry and surface chemical inhomogeneities are observed.

In addition to their remarkable magnetic and chemical surface characteristics, many cooler Ap stars also exhibit high-overtone non-radial acoustic p-mode pulsations. There are more than 30 such rapidly oscillating Ap (roAp) stars known at the present time (Kurtz \& Martinez 2000). These objects oscillate with periods in the range of 6-21 min, while their light variation amplitudes rarely exceed $10 \mathrm{mmag}$ in Johnson $B$. Photometric investigations of roAp stars carried out during the last 25 years have yielded unique asteroseismic information on the internal structure and fundamental parameters of roAp pulsators (e.g., Matthews et al. 1999, Cunha et al. 2003).

The observed pulsation amplitudes of roAp stars are modulated according to the visible magnetic field structure, pointing to a defining role played by magnetic fields in exciting the oscillations and shaping the main pulsation properties. Observation of the coincidence of the magnetic field and pulsation amplitude extrema gave rise to the oblique pulsator model (OPM, Kurtz 1982), which attributes the main characteristics of roAp pulsations to an oblique $\ell=1, m=0$ mode, aligned with the axis of a quasi-dipolar magnetic field. The OPM gave a rather successful geometrical explanation of the main features in the roAp frequency spectra. However, subsequent detailed studies of roAp pulsations have revealed that the mode geometry in some stars defies a simple interpretation in terms of a single spherical harmonic (e.g., Kurtz et al. 1997).

Several theoretical investigations (Bigot \& Dziembowski 2002, Saio \& Gautschy 2004, Saio $2005)$ studied the effects of the distortion of oblique pulsation mode geometry by the global magnetic field and stellar rotation. Bigot \& Dziembowski (2002) suggested that the rotational distortion of pulsation eigenmodes is represented by a superposition of non-axisymmetric 
spherical harmonic components, and that there is no alignment of the pulsation axis and the dipolar magnetic field. On the other hand, Saio \& Gautschy (2004) and Saio (2005) found an axisymmetric pulsation structure aligned with the magnetic field and predicted that the $\ell=1$ mode should be distorted by a dipolar magnetic field in such a way that the pulsation amplitude is strongly confined to the magnetic axis.

Sophistication of these theoretical models notwithstanding, it became clear that modelling of the photometric light curves of roAp stars is unable to provide useful tests of magnetoacoustic theories. The information content of the time-resolved photometric observations is small due to averaging of the pulsational disturbances over the visible stellar hemisphere and is also highly uncertain because rapid light variation in roAp stars involves non-linear and non-adiabatic effects that are poorly understood (Medupe \& Kurtz 1998). The latter problem explains why no consistent physical picture of the photometric variability of roAp has ever been developed. Instead of deducing the structure of the luminosity perturbations from first principles, all attempts to interpret photometric observations of roAp stars have assumed that the luminosity perturbations are proportional to the pulsational displacement. Therefore, constraints on the pulsation mode geometry obtained from the photometry of roAp stars are inherently indirect, which arguably makes any subsequent inferences about the physics of magnetoacoustic oscillations questionable.

\section{Spectroscopic studies of roAp pulsations}

The investigation of pulsational variations in the spectral line profiles of roAp stars observed at high time and spectral resolution provides much more direct and unprecedentedly rich information about the vertical and horizontal structure of $\mathrm{p}$ modes and about their relation to the magnetic field topology, chemical inhomogeneities and anomalous atmospheric structure of Ap stars.

Recently major progress in the observational study of roAp stars was achieved by employing time-series spectroscopy. Time-resolved observations of magnetic pulsators revealed a surprising diversity, not observed in any other type of pulsating stars, in oscillations of different lines (e.g., Kanaan \& Hatzes 1998). Detailed analysis of the bright roAp star $\gamma$ Equ (Kochukhov \& Ryabchikova 2001a) demonstrated that spectroscopic pulsational variability is dominated by the lines of rare-earth ions, especially those of $\mathrm{Pr}$ and $\mathrm{Nd}$. On the other hand, light and iron-peak elements do not pulsate with amplitudes above $50-100 \mathrm{~m} \mathrm{~s}^{-1}$. This is at least an order of magnitude lower than the $\sim 1 \mathrm{~m} \mathrm{~s}^{-1}$ variability observed in the lines of rare-earth elements (REE). Many other roAp stars have been found to show a very similar overall pulsational behaviour (Kochukhov \& Ryabchikova 2001b, Balona 2002, Mkrtichian et al. 2003, Ryabchikova et al. 2007a).

\section{Magnetoacoustic tomography}

The peculiar characteristics of p-mode pulsation in roAp stars were clarified by Ryabchikova et al. (2002), who were the first to relate the pulsational variability to vertical stratification of chemical elements. This study of the atmospheric properties of $\gamma$ Equ showed that the light and iron-peak elements are enhanced in the lower atmospheric layers $\left(\log \tau_{5000} \geq-0.5\right)$, whereas REE ions are concentrated in a cloud with a lower boundary at $\log \tau_{5000} \leq-4$ (Mashonkina et al. 2005). Thus, high-amplitude pulsations observed in REE lines occur in the upper atmosphere, while lines of elements showing no significant variability form in the lower atmosphere. This leads to the following general picture of roAp pulsations: we observe a signature of a magnetoacoustic wave, propagating outwards with increasing amplitude through the chemically stratified atmosphere. 

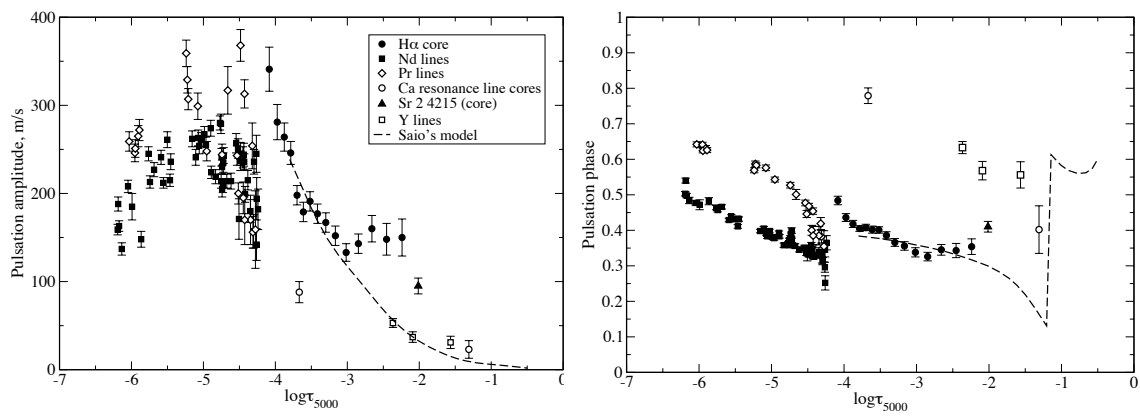

Figure 1: Reconstruction of the vertical cross-section of pulsation mode in the roAp star HD 24712. Symbols show the observed amplitude (left panel) and phase (right panel) of the radial velocity variation for different spectral lines. The dashed line illustrates the theoretical depth dependence of the pulsation wave properties (H. Saio, private communication).

The presence of significant phase shifts between the pulsation radial velocity curves of REEs (Kochukhov \& Ryabchikova 2001a), or even between lines of the same element (Mkrtichian et al. 2003), can be attributed chemical stratification effects and, possibly, to the short vertical wavelength of the running magnetoacoustic wave. These unique properties of roAp pulsations, combined with a presence of large vertical abundance gradients in the line-forming region, make it possible to resolve the vertical structure of $\mathrm{p}$ modes and to study propagation of pulsation waves at the level of detail previously possible only for the Sun.

The study by Ryabchikova et al. (2002) represents the first attempt to use the vertical chemical inhomogeneities as spatial filters which resolve the vertical p-mode structure. The basic idea of this pulsation tomography approach consists of characterizing the pulsational behaviour of a sample of spectral lines and subsequent interpretation of these observations in terms of the pulsation wave propagation. Chemical stratification analysis of REE lines constrains formation depths of pulsating lines, thus allowing one to associate geometrical height with the amplitude and phase of RV pulsations.

Figure 1 illustrates results of the pulsation tomography analysis of the roAp star HD 24712. This star was observed simultaneously by the MOST satellite and from the ground, using highresolution spectrographs at several large telescopes, including the ESO VLT. Using these time-series spectra, Ryabchikova et al. (2007a) studied the pulsational variation of more than 600 lines. Pulsation amplitudes and phases for several characteristic lines of light elements, the core of $\mathrm{H} \alpha$ and numerous REE lines are plotted in Fig. 1 as a function of optical depth. Observations are compared with the theoretical p-mode cross-section $(\mathrm{H}$. Saio, private communication). NLTE line formation was taken into account in chemical stratification analysis of REE ions. This modelling reveals a rapid increase of the pulsation amplitude with height and the respective change of the pulsation phase. The oscillation amplitude reaches maximum at $\log \tau_{5000} \approx-4.5$ and decreases in the higher layers.

Preliminary NLTE stratification analysis of $\operatorname{Pr}$ (Ryabchikova et al. 2007c) suggests that the formation heights of the $\mathrm{Pr}$ III absorption features are not too different from Nd III, despite a clear phase offset between the two groups of lines in HD 24712. This phase difference, as well as the amplitude and phase jump between the uppermost layers probed by the $\mathrm{H} \alpha$ core and the location of the REE cloud, may reflect shortcomings of the complicated NLTE analysis. Alternatively, it is possible that we are seeing effects of the inhomogeneous surface distribution of different REEs in HD 24712. Magnetic Doppler images obtained by Lüftinger et al. (2007) show that the horizontal geometry of the $\mathrm{Pr}$ and $\mathrm{Nd}$ distribution is not the same. This may lead to different pulsational behaviour because the vertical structure of 
magnetoacoustic modes depends on the field strength and orientation (Cunha 2006) and, therefore, may be different at the locations of the Pr and Nd spots in HD 24712.

The detailed pulsation tomography analysis based on NLTE chemical stratification modelling is very demanding in terms of the quality of observations, required input data and computer resources. This is why only two roAp stars, $\gamma$ Equ and HD 24712, were studied with this method up to now. A different approach to the pulsation tomography problem was proposed by Ryabchikova et al. (2007b). They noted that in the framework of the outward propagating magnetoacoustic wave one expects a continuous amplitude vs. phase relation for pulsation modes. The amplitude-phase diagrams offer a possibility to trace the vertical variation in the mode structure without assigning physical depth to pulsation measurements. Ryabchikova et al. (2007b) analysed a sample of ten roAp stars, measuring variations of several hundred lines for each object. The amplitude-phase diagrams were constructed for each star and the resultant vertical mode cross-sections were compared with other pulsational characteristics and with the fundamental stellar parameters. As an outcome of this analysis, it was discovered that the form of pulsational perturbation changes from a predominantly standing to a mainly running wave within the REE line-forming region. It appears that the location of this interesting modification of the pulsation wave properties shifts towards higher layers for cooler roAp stars.

\section{Variability of line bisectors}

In addition to the diversity in the pulsation signatures of different elements and ions, the variation of individual strong REE lines in roAp stars is far from trivial. The most surprising observation is a large change in the amplitude and phase of bisector RV with intensity inside individual lines. In some sharp-lined roAp stars the RV amplitude increases from a few hundred $\mathrm{km} \mathrm{s}^{-1}$ to several $\mathrm{km} \mathrm{s}^{-1}$ as one moves towards the outer parts of the line profiles. At the same time, the pulsation phase shows complicated trends with bisector intensity, sometimes changing by up to $180^{\circ}$. This remarkable bisector variation was first discovered in $\gamma$ Equ (Sachkov et al. 2004) and has been demonstrated for other roAp stars (Kurtz et al. 2005, Ryabchikova et al. 2007ab). The rapidly rotating pulsators, such as HD 99563 (Elkin et al. 2005), show an increase of bisector amplitude towards the core - a trend opposite to that of $\gamma$ Equ.

The line core and wings are expected to sample somewhat different parts of the atmosphere and this is why changes in the bisector variation across individual lines are often attributed to height effects. According to this explanation, modification of bisector oscillations indicates a remarkably complex and rapidly changing pulsation mode structure, with several nodes located in the line-forming region. However, this interpretation must be viewed with caution. A rapid change of the pulsation wave properties with height is inconsistent with the welldefined, smooth amplitude and phase depth dependence inferred by pulsation tomography. Theoretical models (Saio \& Gautschy 2004, Saio 2005) also predict no nodes in the upper atmosphere. Moreover, the implicit assumption that any deviation from a constant amplitude and phase of the bisector should be interpreted as a height effect is questionable. In fact, no studies looked at the bisector behaviour in normal non-radial pulsators. It appears that at least part of the core-to-wing change of the bisector amplitude may be ascribed to the presence of a high- $\ell$ harmonic contribution in the horizontal pulsation structure.

Interpretation of the bisector variability is even more ambiguous for rapidly rotating roAp stars. In these objects the oblique non-radial pulsations are superimposed onto the much larger velocity field due to the stellar rotation. The primary consequence of the dominant rotational broadening of spectral lines is that the mapping between bisector intensity and atmospheric height is no longer valid. Instead, the spectral line wings are formed close to the limb of the visible stellar disk, whereas the line core region is primarily sensitive to the disk centre. Detailed spectrum synthesis calculations demonstrate that in the presence of chemical 
spots one can easily obtain substantial core-to-wing changes of the bisector amplitude and phase without any depth dependence of pulsation wave. Therefore, the interpretation of the bisector variation of rapidly rotating roAp stars in terms of vertical structure of $p$ modes (e.g., Elkin et al. 2005) is probably incorrect.

\section{Pulsation Doppler imaging}

The outstanding pulsational variability of REE lines in rapidly rotating roAp stars permits detailed mapping of the horizontal structure of pulsations. The oblique nature of non-radial oscillations allows pulsational monitoring from different aspect angles, thus facilitating reconstruction of the pulsation pattern. Using this unique geometrical property of roAp pulsations, Kochukhov (2006) has carried out high-resolution spectroscopic monitoring of the prototype roAp star HD 83368 (HR 3831). This star was observed at the ESO 3.6-m telescope during six nights over a period of two weeks. Full rotational phase coverage with the time-resolved spectra was obtained, supplying observational material for the first comprehensive investigation of the pulsational line profile variability (LPV) in a roAp star.

The moderately rapid ( $v_{\mathrm{e}} \sin i=33 \mathrm{~km} \mathrm{~s}^{-1}$ ) rotation of HD 83368 allows to use the Doppler effect in the spectral lines to resolve both the horizontal topology of chemical inhomogeneities and velocity perturbations due to non-radial oscillations. Kochukhov (2004a) extended the principles of Doppler imaging (DI) to the reconstruction of the time-dependent velocity field. In this approach the surface pulsation velocity amplitudes are recovered directly from the observed line profile variability, without a priori constraints on the functional form of pulsation maps. This makes pulsation DI one of few tools suitable for addressing the daring task of inferring non-radial pulsation patterns distorted by a magnetic field and stellar rotation.

Applying the pulsation mapping technique to the roAp star HD 83368, Kochukhov (2004b) obtained the first stellar Doppler image of the velocity field. DI analysis of HD 83368 revealed a nearly axisymmetric pulsation geometry and for the first time independently confirmed the alignment of the non-radial pulsation and the magnetic field. Pulsation mapping finds the oblique pulsator geometry as a result of the assumption-free analysis, in contrast to all previous studies of roAp stars which started from the assumption that the OPM is valid. Highresolution maps of pulsations in HD 83368 were used by Kochukhov (2004b) to disentangle different harmonic contributions to the pulsation geometry. It was shown that the oscillations are shaped as suggested by Saio \& Gautschy (2004), whereas the non-axisymmetric pulsation components predicted by the theory of Bigot \& Dziembowski (2002) cannot be detected. This demonstrates a dominant role of the magnetic perturbation of the $\mathrm{p}$ modes and a considerably less important influence of stellar rotation.

\section{Rapid line profile variation in sharp-lined roAp stars}

Despite dramatic progress in understanding the vertical structure of pulsation modes in slowly rotating roAp stars, relatively little attention has been paid to the problem of inferring the horizontal geometry of pulsations. It is often assumed that a horizontal cross-section of non-radial pulsation is given by an oblique axisymmetric mode of low degree, similar to the pulsation geometries found for rapidly rotating roAp stars. Thus, the question of systematic mode identification has not been thoroughly investigated in the case of sharp-lined magnetic pulsators, which represent the majority of roAp stars.

Understanding rapid LPV of slowly rotating roAp stars turns out to be a challenging task. The first observation of roAp line profile variability (Kochukhov \& Ryabchikova 2001a) demonstrated the presence of unusual blue-to-red running features in the residual spectra of $\gamma$ Equ. Moreover, a single-wave variability of the REE line width in this star is clearly inconsis- 


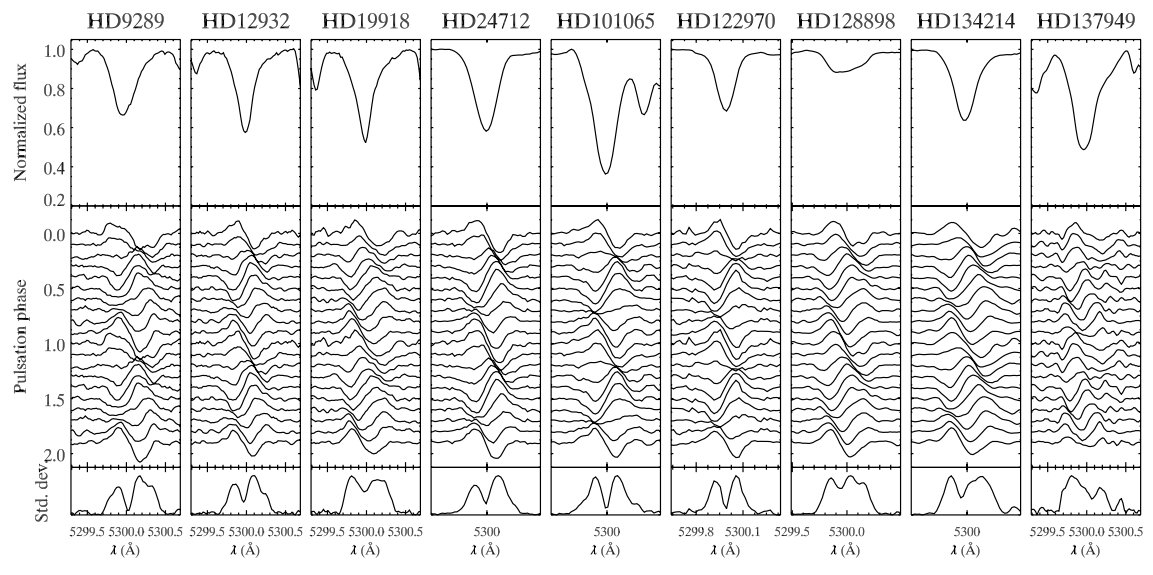

Figure 2: Profile variations of the $\operatorname{Pr}$ III $5300 \AA$ line in the spectra of sharp-lined roAp stars. The average spectrum is plotted in the upper part of each panel. The time-series of the difference spectra is shown in the middle. The bottom curve presents the wavelength dependence of the standard deviation.

tent with any axisymmetric pulsation geometry described by spherical harmonics (Aerts et al. 1992, Kochukhov 2005). This led Kochukhov \& Ryabchikova (2001a) to speculate about the possible presence of non-axisymmetric modes in $\gamma$ Equ - a suggestion equivalent to stating that the classical OPM is not applicable to this star. Later Shibahashi et al. (2004) argued that the blue-to-red running waves in the residual spectra of $\gamma$ Equ are inconsistent with spectral variability expected for any, axisymmetric and non-axisymmetric alike, low-degree modes.

The puzzle of the pulsational LPV in sharp-lined roAp stars has been solved by Kochukhov et al. (2007). This study presented a comprehensive survey of profile variability in ten roAp stars using observations obtained at the VLT and CFHT telescopes. The variations of the REE lines were investigated in detail and a prominent change of the profile variability pattern with height was discovered for all roAp stars. The profile variability of at least one rare-earth ion in each investigated star is characterized by the blue-to-red moving features, previously discovered in $\gamma$ Equ. Figure 2 shows an example of this interesting behaviour, common in rapidly rotating non-radial pulsators, but completely inexplicable in the framework of the standard OPM of slowly rotating roAp stars.

The analysis of the line profile moments and spectrum synthesis calculations presented by Kochukhov et al. (2007) demonstrates that unusual oscillations in spectral lines of roAp stars arise from the pulsational modulation of line widths. This variation occurs approximately in quadrature with the radial velocity changes, and its amplitude rapidly increases with height in the stellar atmosphere. Kochukhov et al. (2007) proposed that the line width modulation is a consequence of the periodic expansion and compression of turbulent layers in the upper atmospheres of roAp stars. This means that the line profile changes observed in slowly rotating magnetic pulsators should be interpreted as a superposition of two types of variability: the usual time-dependent velocity field due to an oblique low-order pulsation mode and an additional line width modulation, synchronized with the changes of stellar radius. Figure 3 shows that this new OPM correctly reproduces the main features in the observed pulsational variability of line profiles and moments in roAp stars. 

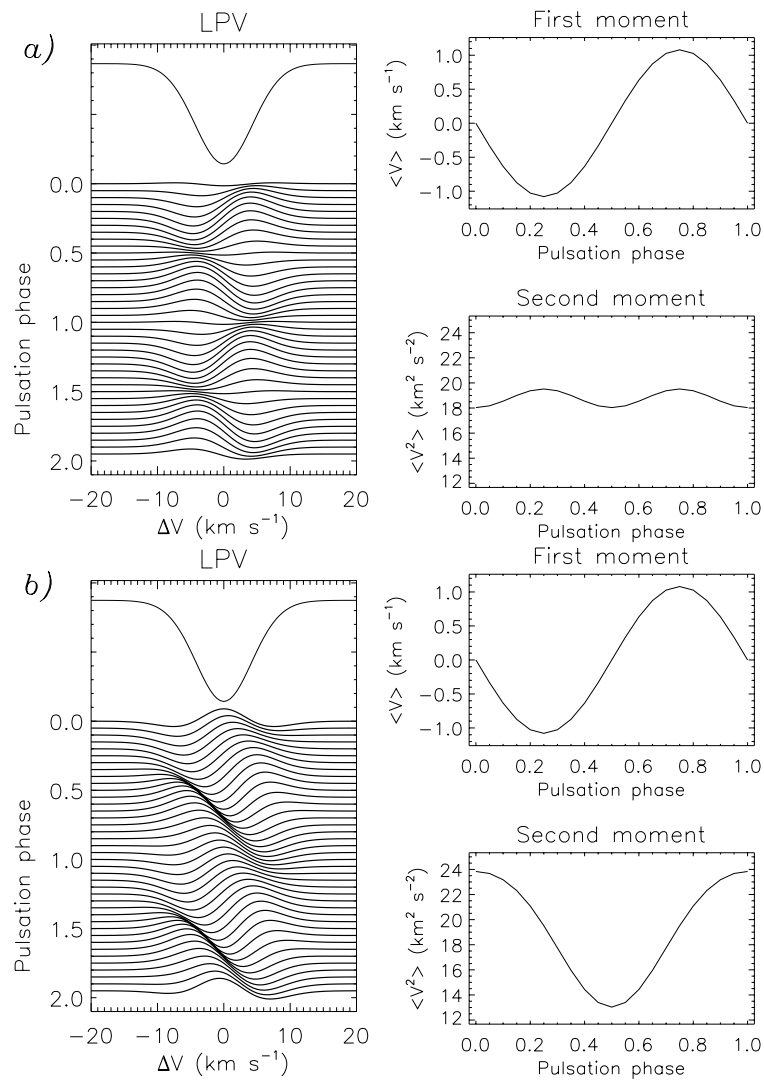

Figure 3: Line profile variations of an oblique non-radial pulsator. a) Spectrum variability for the $\ell=1$, $m=0$ mode viewed from the pulsation pole. b) Effect of adding harmonic variability of the line width. In each panel the left plot shows the average line profile on top and a time series of the difference spectra below. The right panels illustrate variation of the first (RV, upper plot) and second (line width, lower plot) line profile moments.

\section{Conclusions and outlook}

Recent investigations of the spectroscopic variability of roAp stars revealed an interesting and complex picture. The most prominent effect, distinguishing roAp stars from all other pulsators, is the close interrelation between the chemical stratification and pulsational variability. Magnetoacoustic waves pass through distinct chemical clouds in the upper atmospheric layers, giving rise to depth-dependent amplitude and phase shifts in the radial velocity variation of different elements.

The quality of observational material and available computing resources have reached the stage when it becomes feasible to address the task of constructing 3-D dynamical models of pulsating stellar atmospheres. The pulsation Doppler imaging and magnetoacoustic tomography techniques can be combined in a self-consistent remote sensing procedure, aimed at the recovery of the 3-D geometry of pulsations and chemical inhomogeneities. This can be 
achieved by applying the pulsation Doppler imaging method to the time-series observations of spectral lines formed at different heights and then combining the resulting horizontal slices of the pulsation pattern into a 3-D velocity field map.

Construction of the empirical maps should be supported by the advanced theoretical modelling of peculiar-star atmospheres and pulsations. In particular, a realistic study of the interaction between pulsations, turbulence and magnetic field in the tenuous outer layers of roAp atmospheres is urgently needed to clarify many puzzling aspects of the spectroscopic variation of roAp stars.

Acknowledgments. I thank the organizers of the Vienna Workshop on the Future of Asteroseismology for inviting me to present this review. My participation in the workshop was supported by the grants from the Swedish Kungliga Fysiografiska Sällskapet and the Royal Academy of Sciences.

\section{References}

Aerts C., De Pauw M., Waelkens C., 1992, A\&A, 266, 294

Balona L. A., 2002, MNRAS, 337, 1059

Bigot L., Dziembowski W. A., 2002, A\&A, 391, 235

Cunha M. S., 2006, MNRAS, 365, 153

Cunha M. S., Fernandes J. M. M. B., Monteiro M. J. P. F. G., 2003, MNRAS, 343, 831

Elkin V. G., Kurtz D. W., Mathys G., 2005, MNRAS, 364, 864

Kanaan A., Hatzes A. P., 1998, ApJ, 503, 848

Kochukhov O., 2004a, A\&A, 423, 613

Kochukhov O., 2004b, ApJ, 615, L149

Kochukhov O., 2005, A\&A, 438, 219

Kochukhov O., 2006, A\&A, 446, 1051

Kochukhov O., Ryabchikova T., 2001a, A\&A, 374, 615

Kochukhov O., Ryabchikova T., 2001b, A\&A, 377, L22

Kochukhov O., Ryabchikova T., Weiss W. W., Landstreet J. D., Lyashko D., 2007, MNRAS, in press

Kurtz D. W., 1982, MNRAS, 200, 807

Kurtz D. W., van Wyk F., Roberts G., et al., 1997, MNRAS, 287, 69

Kurtz D. W., Martinez P., 2000, Baltic Astron., 9, 253

Kurtz D. W., Elkin V. G., Mathys G., 2005, MNRAS, 358, L6

Lüftinger T., Kochukhov O., Ryabchikova T., Weiss W. W., Ilyin I., 2007, Comm. Asteroseis., 150, 85

Mashonkina L., Ryabchikova T., Ryabtsev A., 2005, A\&A, 441, 309

Matthews J. M., Kurtz D. W., Martinez P., 1999, ApJ, 511, 422

Medupe T., Kurtz D. W., 1998, MNRAS, 299, 371

Mkrtichian D. E., Hatzes A. P., Kanaan A., 2003, MNRAS, 345, 781

Ryabchikova T., Piskunov N., Kochukhov O., et al., 2002, A\&A, 384, 545

Ryabchikova T., Sachkov M., Weiss W. W., et al., 2007a, A\&A, 462, 1103

Ryabchikova T., Sachkov M., Kochukhov O., Lyashko D., 2007b, A\&A, submitted

Ryabchikova T., Mashonkina L., Ryabtsev A., Kildiyarova R., Khristoforova M., 2007c, Comm. Asteroseis., 150, 83

Sachkov M., Ryabchikova T., Kochukhov O., et al., 2004, in Kurtz D. W., Pollard K. R., eds, ASP Conf. Ser. Vol. 310, Variable Stars in the Local Group. San Francisco, p. 208

Saio H., 2005, MNRAS, 360, 1022

Saio H., Gautschy A., 2004, MNRAS, 350, 485

Shibahashi H., Kurtz D. W., Kambe E., Gough D. O., 2004, in Zverko J., Žižňovský J., Adelman S. J., Weiss W. W., eds, IAU Symp. 224, The A-star Puzzle. Cambridge Univ. Press, p. 829 


\section{DISCUSSION}

Kepler: The assumption of a single spherical harmonic is wrong in terms of the pulsation, i.e. the real change in the star. You need sums of spherical harmonics or something similar.

Kochukhov: Yes, this is precisely what happens. The pulsations are distorted by the magnetic fields and therefore they are not described by a single spherical harmonic, and this is why we are not using spherical harmonics. We are reconstructing the pulsations making no a priori assumptions about horizontal structure, just like in other applications of Doppler imaging.

Metcalfe: When you do these reconstructions via Doppler imaging for multiple lines, do you derive the same inclination angles for the magnetic axis?

Kochukhov: The magnetic axis is not constrained by the intensity spectra used for pulsation mapping; it is constrained by the analysis of polarimetric stellar measurements. Doppler images discussed here yield inclination of the pulsation axis, which is found to coincide with the orientation of the magnetic axis inferred in the previous studies of the star. As for multiline analysis, it is not yet feasible because the spectra available to us cover only a short wavelength region.

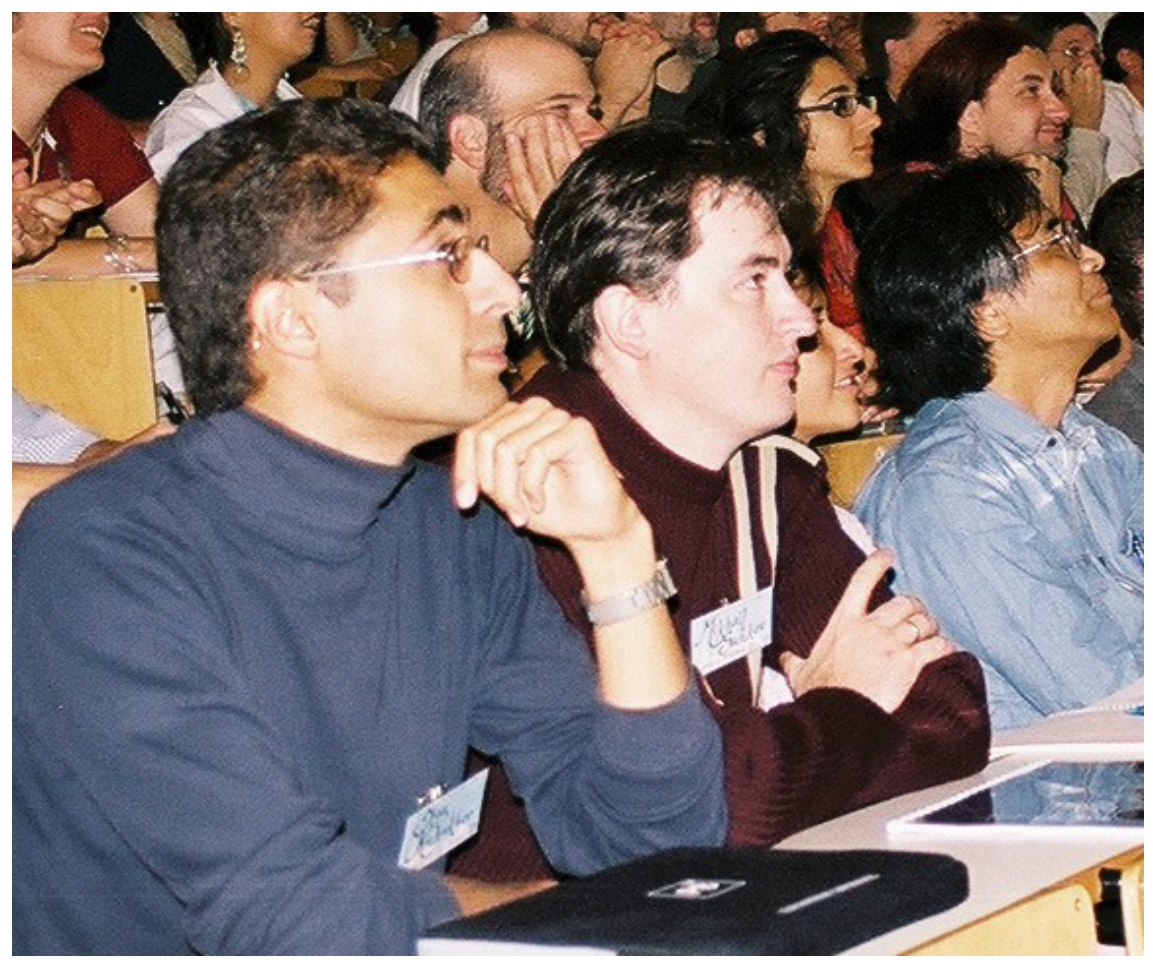

Oleg Kochukhov and Mikhail Sachkov concentrating on a discussion. 